\title{
Physicochemical characteristics, nutritional composition, and phytochemical profiles of nine Algerian date palm fruit (Phoenix dactylifera L.) varieties
}

\begin{abstract}
Nine varieties of Algerian date palm fruits (Phoenix dactylifera L.) including Lahmira, Timjouhert, Adham Talmine, Deglet Talmine, Adam Boullah, Tinasser, Deglet, Deglet Nour, and Takerbouch were distinguished via Proton Nuclear Magnetic Resonance (1H-NMR) metabolomics approach. The quality was evaluated based on the metabolite composition, proximate analysis, physicochemical characteristics, and biological activities which include nitric oxide (NO) inhibition via the cell-based approach and NO scavenging abilities. A total of 49 metabolites were tentatively characterized including amino acids, organic acids, and phenolic compounds. The Principal Component Analysis and Hierarchical Clustering Analysis revealed a consistent grouping of Deglet, Takerbouch, and Deglet Noor from the rest of six varieties. Among the metabolites that contributed toward the observed segregation were serine, glycine, glucose, fructose, and sucrose. With regard to metabolite composition, NO scavenging activities, total soluble solids, and proximate analysis, Deglet dates displayed a better quality as compared to the other Algerian dates.
\end{abstract}

Keyword: Algerian date fruits; Date palm fruit varieties; NMR metabolomics; Phoenix dactylifera; RAW 264.7 cells 